\title{
Surface-Coated CdS Nanocrystals
}

\author{
J.R.L. Fernandez ${ }^{a}$, M. DE SOuzA-PARISE ${ }^{b}$ And P.C. Morais ${ }^{b, c}$ \\ ${ }^{a}$ Universidade Estadual de Santa Cruz, Campus Soane Nazaré de Andrade, Ilhéus BA 45662-900, Brazil \\ ${ }^{b}$ Universidade de Brasília, Instituto de Física, Núcleo de Física Aplicada, Brasília DF 70910-900, Brazil \\ ${ }^{c}$ Huazhong University of Science and Technology, School of Automation, Wuhan 430074, China
}

(Received January 2, 2013; in final form April 20, 2013)

\begin{abstract}
CdS nanocrystals were prepared using a standard colloidal route (co-precipitation chemical reaction) whereas modulation of the optical and structural properties was realized via surface coating the nanocrystals with ethylene glycol molecules using an in situ procedure. The as-produced nanocrystals were characterized by X-ray diffraction, Raman spectroscopy, UV-absorption measurements and photoluminescence. The nanocrystal quality was revealed by the spectroscopic data, though the final product presents a mixture of both zinc blende (cubic) and wurtzite (hexagonal) phases. The X-ray data indicated that the concentration of the ethylene glycol used during the synthesis process modulates the nanocrystal size and the crystal phase of the end material.
\end{abstract}

DOI: $10.12693 /$ APhysPolA.124.108

PACS: 78.30.Fs, 78.40.Fy, 78.55.Et, 78.67.Bf, 78.70.Ck

\section{Introduction}

The recent years have been witness to a huge development in the synthesis and in the investigation of the physical properties of nanocrystals, mostly driven by the possibilities of medical and industrial applications [1-3]. Due to this, the understanding of the mechanisms which control the phase stability are key issues. The phase stability for instance depends on the temperature, pressure, nanocrystal size, and surfactant [4-6]. Studies regarding the growth of CdS nanocrystals using different methods have been carried out, emphasizing the effect of the annealing temperature on the phase transformation from the zinc blende to the wurtzite structure $[7,8]$. In addition, there are studies reporting the influence of the surfactants on the phase stability [9]. Moreover, the literature reports that the parameters which influence the phase stability also influence the shape of the nanocrystal, giving rise to materials with modified optical and electrical properties [10-12]. In this study, we report on the structural and optical properties of CdS nanocrystals grown in the absence (uncapped) and in the presence (capped) of ethylene glycol at different concentrations. Ethylene glycol was selected due to be a surface-stabilizing agent. Also, in the literature it was reported that it is used for nanoparticle suspension (nanofluid) and as solvent in the hydrothermal synthesis due to its ability to reduce metal salts to lower oxidation states and its high boiling point $[13,14]$.

\section{Sample preparation and experimental techniques}

Analytic grade cadmium nitrate and ammonium sulfide reagents were used as received without further purification. For the surface-uncapped CdS nanocrystal (REF1, REF2) synthesis $1.0 \mathrm{M}$ aqueous solutions of $\left(\mathrm{NH}_{4}\right)_{2} \mathrm{~S}$ and $\mathrm{Cd}\left(\mathrm{NO}_{3}\right)_{2}$ were prepared separately. Co-precipitation chemical reactions were carried out mixing equal volumes of the as-prepared aqueous solutions.
Surface-uncapped CdS nanocrystal was precipitated at room temperature and under mechanical stirring. After precipitation the samples were centrifuged, washed with deionized water, and dried in vacuum at $10^{-3}$ mbar. For the surface-capped samples, the co-precipitation process was modified by adding different amount of ethylene glycol to the $\left(\mathrm{NH}_{4}\right)_{2} \mathrm{~S}$ aqueous solutions $(10 \%, 20 \%$ and $50 \%$ ) (see Table I).

TABLE I

Growth conditions of the samples.

\begin{tabular}{c|c|c}
\hline \hline Sample & $T[\mathrm{~K}]$ & Comments \\
\hline REF1 & 300 & $0.5 \mathrm{M}$ of $\mathrm{Cd}\left(\mathrm{NO}_{3}\right)$ \\
REF2 & 300 & $1.0 \mathrm{M} \mathrm{of} \mathrm{Cd}\left(\mathrm{NO}_{3}\right)$ \\
ETG1 & 300 & ethylene glycol $(10 \%)$ \\
ETG2 & 380 & ethylene glycol $(10 \%)$ \\
ETG3 & 300 & ethylene glycol $(20 \%)$ \\
ETG4 & 300 & ethylene glycol $(50 \%)$
\end{tabular}

$\mathrm{X}$-ray diffractograms (XRD) of the samples were recorded using a Shimadzu (XRD-6000) system equipped with a $\mathrm{Cu} K_{\alpha}$ radiation source. Optical absorption measurements were performed using a Varian spectrophotometer (Carry 500 Scan-UV-VIS-NIR). Room-temperature micro-Raman measurements were carried out using a commercial triple spectrometer (Jobin-Yvon model T64000) equipped with a CCD detector. The Raman system was calibrated using the silicon Raman peak centered at $521 \mathrm{~cm}^{-1}$. Photoluminescence measurements were performed using the Raman setup equipped with a GaAs photomultiplier detector. The laser line $(488 \mathrm{~nm})$ from an argon ion laser was used to optically excite the samples.

\section{Results and discussion}

Figure 1 shows the XRD of the samples (REF1, REF2, ETG1, ETG2, ETG3, and ETG4). The XRD spec- 
tra suggest that the samples are crystalline. Samples obtained with low ethylene-glycol concentration have hexagonal phase and samples grown with high ethylene-glycol concentration have both phases (cubic and hexagonal), with cubic phase tendency. We claim this because the XRD of the samples (ETG1 and ETG2) are similar and both show peaks at $24.8^{\circ}, 26.4^{\circ}, 28.2^{\circ}, 36.5^{\circ}, 43.8^{\circ}$, $47.8^{\circ}$, and $51.8^{\circ}$, corresponding to X-ray reflections from (100), (002), (101), (102), (110), (103) and (112) planes associated to $\mathrm{CdS}$ wurtzite phase (hexagonal). The samples (ETG3 and ETG4) show, instead, X-ray reflection peaks at $26.4^{\circ}, 43.8^{\circ}$, and $51.8^{\circ}$, which related to the reflection (111), (220) and (311) planes from the CdS zinc blende phase (cubic). The samples (ETG3 and ETG4) also revealed weak X-ray diffraction peaks corresponding to the reflections from (100) and (101) planes, typical of the hexagonal phase (like to the uncapped samples REF1 and REF2).

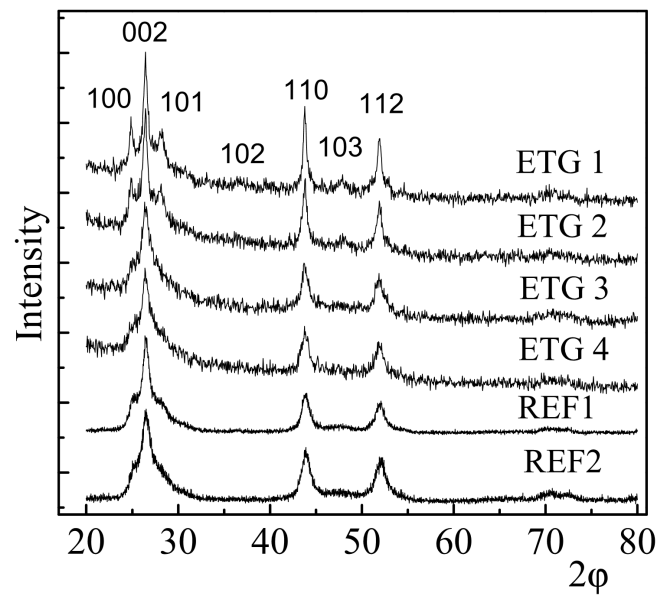

Fig. 1. X-ray diffraction spectra of the samples.

In order to check more accurately the presence of two CdS phases (hexagonal and cubic) the XRD of the samples were fitted. Lorentzian functions were successfully used to fit the samples, indicating the occurrence of the hexagonal phase in all of them. From the values of FWHM of the XRD peaks, the particle size was calculated as $8.9 \mathrm{~nm}$ (REF1), $7.0 \mathrm{~nm}$ (REF2), $13.7 \mathrm{~nm}$ (ETG1), $12.6 \mathrm{~nm}$ (ETG2), $8.2 \mathrm{~nm}$ (ETG3), and $9.6 \mathrm{~nm}$ (ETG4). The crystallite size was calculated using the Debye-Scherrer formula for the (002) reflection plane of XRD spectra. Samples grown with lower ethylene glycol concentration (ETG1 and ETG2) have hexagonal phase and bigger nanoparticle size when compared to the other samples.

Our findings are in very good agreement with the report by Singh et al. [15] which obtained critical surfactant concentration of $10 \mathrm{mM}$ cetyl trimethyl ammonium bromide. Above this value the authors reported a phase transformation (from hexagonal phase to cubic phase) and the decrease of the particle sizes. In our case we claim that as the ethylene glycol concentration increases the growth velocity is reduced. This reduction could be due to high surfactant concentration at the nanoparticle surface [15]. Also, higher surfactant concentration around the nanoparticle should delimit the $\mathrm{Cd}^{2+}$ quantity that arrives to the nanoparticle surface.

$\mathrm{CdS}$ grown with hexagonal phase has six Raman active modes denoted as E2 at $43 \mathrm{~cm}^{-1}, \mathrm{~A} 1$ (TO) at $234 \mathrm{~cm}^{-1}$, E1 (TO) at $243 \mathrm{~cm}^{-1}, \mathrm{E} 2$ at $256 \mathrm{~cm}^{-1}, \mathrm{~A} 1$ (LO) at $305 \mathrm{~cm}^{-1}$, and $\mathrm{E} 1$ (LO) at $307 \mathrm{~cm}^{-1}$ [16]. CdS grown in cubic phase has the active modes E1 (TO) at $243 \mathrm{~cm}^{-1}$ and A1 (LO) at $305 \mathrm{~cm}^{-1}$.

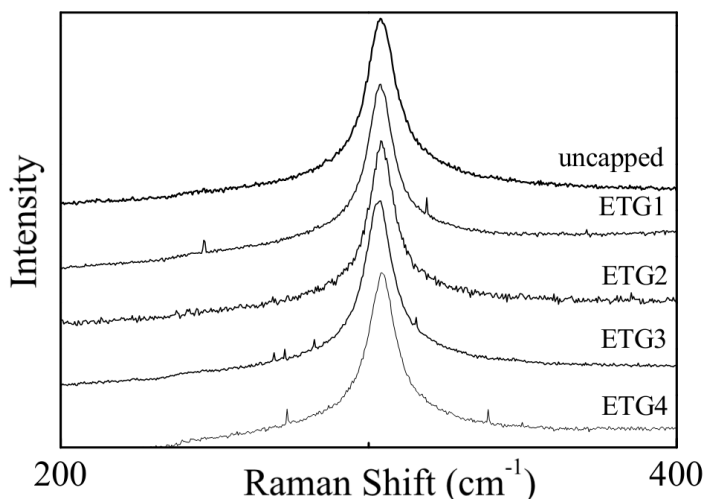

Fig. 2. Raman spectra of the REF2, ETG1, ETG2, ETG3, and ETG4 samples. The spectra were recorded with laser power of $1 \mathrm{~mW}$.

Figure 2 shows the Raman spectra of the samples (REF2, ETG1, ETG2, ETG3, and ETG4). The samples have a Raman peak (LO) around $304 \mathrm{~cm}$. The LO Raman peak of all samples have FWHM in the range of 10 to $12 \mathrm{~cm}^{-1}$, indicating a good crystalline quality, as quoted in the literature [16-18]. Our Raman measurements did not obtain any relation between particle size [19], surface [20] or strain [21]. There was observed only a dislocation of the Raman peak when the samples were measured as a function of laser power.

Figure 3 shows the Raman spectrum (normalized) of the uncapped samples as a function of the laser power. From the figure there is observed a dislocation of the Raman peak from $304 \mathrm{~cm}^{-1}(0.1 \mathrm{~mW})$ to $300 \mathrm{~cm}^{-1}$ $(16 \mathrm{~mW})$. Also the linewidth increased from $10 \mathrm{~cm}^{-1}$ to $22 \mathrm{~cm}^{-1}$. Further the Raman peak intensity is a function of the laser power and begins to decrease when the laser power of $10 \mathrm{~mW}$ is exceeded. This behavior is typical of phase transformation. Sample oxidation could be the origin of the peak dislocation and linewidth (Table II).

Figure 4 shows the optical absorption and photoluminescence spectrum (PL) of the ETG4 sample, at $T=$ $300 \mathrm{~K}$. The peak-like shape of the absorption spectrum indicates that the sample has high oscillator strength value, meaning the presence of localized states. Exciton peaks plus the continuum responds for the absorption spectrum of the size polydispersed sample. Due to the complexity introduced by the size dispersity, the absorption curve was fitted with a convolution of Gaussian func- 


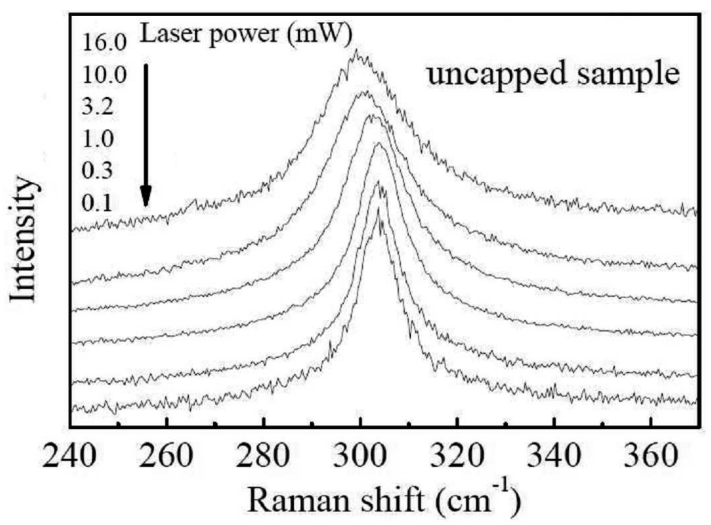

Fig. 3. Behavior of the Raman LO peak (uncapped) as a function of laser power.

\section{TABLE II}

LO peak position and linewidth as a function of the laser power.

\begin{tabular}{c|c|c}
\hline $\begin{array}{c}\text { Laser power } \\
{[\mathrm{mW}]}\end{array}$ & $\begin{array}{c}\text { LO } \\
{\left[\mathrm{cm}^{-1}\right]}\end{array}$ & $\begin{array}{c}\text { Linewidth } \\
{\left[\mathrm{cm}^{-1}\right]}\end{array}$ \\
\hline 1 & 303.6 & 9.7 \\
3 & 303.6 & 9.7 \\
10 & 303.9 & 10.8 \\
32 & 302.8 & 14.7 \\
100 & 300.9 & 19.0 \\
160 & 299.9 & 21.7
\end{tabular}

tions. The peak positions obtained from this fitting and associated to the three components are shown in Fig. 4. We claim that the absorption peaks at 2.377 and $2.488 \mathrm{eV}$ are related to the cubic and hexagonal phase of $\mathrm{CdS}$, respectively. These values are blue-shifted when compared with the $300 \mathrm{~K}$ values of 2.352 (cubic) and $2.452 \mathrm{eV}$ (hexagonal). Calculation of the exciton absorption energy was performed assuming that the exciton binding energy is $28 \mathrm{meV}$ for both phases [22, 23] and assuming the band edge values of $2.38 \mathrm{eV}$ and $2.48 \mathrm{eV}$ for the cubic [24] and hexagonal [22, 25] phases, respectively.

To calculate the diameter of the nanocrystal based on the absorption spectrum the empirical formula [26] is used:

$$
D[\mathrm{~nm}]=-A \lambda^{3}+B \lambda^{2}-C \lambda+13.29,
$$

where $D$ is the nanocrystal diameter (in $\mathrm{nm}$ ), $\lambda$ is the first absorption peak (in nm), $A=6.6521 \times 10^{-8}, B=$ $1.9557 \times 10^{-4}$, and $C=9.2352 \times 10^{-2}$. Using the equation we found $8.87 \mathrm{~nm}$ for the nanocrystal size associated to the CdS cubic phase. This value is in very good agreement with the value that we have obtained from the XRD data.

\section{Conclusion}

The present study revealed that optical and structural properties of nanosized $\mathrm{CdS}$ can be modulated

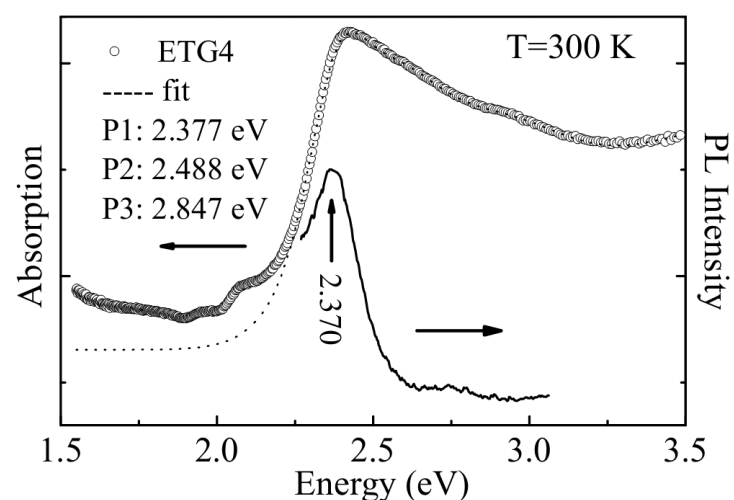

Fig. 4. Optical absorption and PL spectrum of the ETG4 sample. Also there are shown the peak positions obtained from the curve fit.

by coating the nanocrystal surface using ethylene glycol molecules. Standard co-precipitation chemical reaction in aqueous media containing the starting reagents $\left(\mathrm{Cd}^{2+}\right.$ and $\left.\mathrm{S}^{2-}\right)$ plus a controlled concentration of the surface coating molecule (ethylene glycol) was successfully used to produce different $\mathrm{CdS}$ nanocrystal samples. Spectroscopic characterization of the as-produced samples confirm the effect of the ethylene glycol molecules added to the reaction media during the production of the CdS nanocrystals. From the analysis of the spectroscopic data we hypothesized that the main role played by the ethylene glycol molecules is reducing CdS surface traps. Finally, the optical measurements revealed that high quality CdS nanocrystals were produced by this chemical route, though a mixture of wurtzite (hexagonal phase) and zinc blende (cubic phase) were found in the end material.

\section{Acknowledgments}

J.R.L. Fernandez is supported by $\mathrm{CNPq}$ process number $503533 / 2003-3$.

\section{References}

[1] V. Biju, T. Itoh, A. Anas, A. Sujith, M. Ishikawa, Anal. Bioanal. Chem. 391, 2469 (2008).

[2] R. Hao, R. Xing, Z. Xu, Y. Hou, S. Gao, S. Sun, Adv. Mater. 22, 2729 (2010).

[3] Y.R. Smith, V. Subramanian, J. Phys. Chem. C 115, 8376 (2011).

[4] C. Ricolleau, L. Audinet, M. Gandais, T. Gacoin, Eur. Phys. J. D 9, (1) 565 (1999).

[5] Y. Xiong, J. Zhang, F. Huang, G. Ren, W. Liu, D. Li, C. Wang, Z. Lin, J. Phys. Chem. C 112, 9229 (2008).

[6] X. Li, H. Zhang, J. Gao, D. Guo, C. Yang, L. Xu, B. Liu, X. Zhang, J. Nanopart. Res. 13, 6563 (2011).

[7] M. Ichimura, F. Goto, E. Arai, J. Appl. Phys. 85, 7411 (1999).

[8] S. Mishra, A. Ingale, U.N. Roy, A. Gupta, Thin Solid Films 516, 91 (2007). 
[9] A. Priyam, A. Chatterjee, S.C. Bhattacharya, A. Saha, J. Cryst. Growth 304, 416 (2007).

[10] H. Chu, X. Li, G. Chen, W. Zhou, Y. Zhang, Z. Jin, J. Xu, Y. Li, Cryst. Growth Des. 5, 1801 (2005).

[11] D. Tari, M. De Giorgi, F.D. Sala, L. Carbone, R. Krahne, L. Manna, R. Cingolani, S. Kudera, W.J. Parak, Appl. Phys. Lett. 87, 224101 (2005).

[12] W. Qingqing, X. Gang, H. Gaorong, Cryst. Growth Des. 6, 1776 (2006).

[13] J. Tavares, S. Coulombe, Powder Technol. 210, 132 (2011).

[14] P.K. Khanna, R.M. Gorte, R. Gokhale, Mater. Lett. 58, 966 (2004).

[15] V. Singh, P.K. Sharma, P. Chauhan, Mater. Chem. Phys. 121, 202 (2010).

[16] K. Palmö, B. Mannfors, L.-O. Pietila, J. Mol. Struct. 174, 101 (1988).

[17] H. Cao, G. Wang, S. Zhang, X. Zhang, D. Rabinovich, Inorg. Chem. 45, 5103 (2006).

[18] M.I. Vasilevskiy, A.G. Rolo, M.J.M. Gomes, O.V. Vikhrova, C. Ricolleau, J. Phys., Condens. Matter 13, 3491 (2001).
[19] A. Imada, S. Ozaki, S. Adachi, J. Appl. Phys. 92, 1793 (2002)

[20] P. Kumar, N. Saxena, F. Singh, A. Agarwal, Physica B 407, 3347 (2012).

[21] B. Schreder, C. Dem, M. Schmitt, A. Materny, W. Kiefer, U. Winkler, E. Umbach, J. Raman Spectrosc. 34, 100 (2003).

[22] M. Montazeri, L.M. Smith, H.E. Jackson, J.M.Y. Rice, Y.J. Choi, J.G. Park, Appl. Phys. Lett. 95, 083105 (2009).

[23] D.G. Thomas, J.J. Hopfield, Phys. Rev. 116, 573 (1959).

[24] D.J. Kim, Y.M. Yu, J.W. Lee, Y.D. Choi, Appl. Surf. Sci. 254, 7522 (2008).

[25] S. Seto, Jpn. J. Appl. Phys. 44, 5913 (2005).

[26] W.W. Yu, L. Qu, W. Guo, X. Peng, Chem. Mater. 15, 2854 (2003). 